\title{
Serum Unconjugated Bilirubin as a Predictor of Specific Cellular Immunity Response in Kidney Transplant Recipients after Administration of SARS- CoV-2 Inactivated Vaccine
}

\section{Lei Zhang}

Jiaqing Yang

Changchun Lai

Min Deng

Xuanying Deng

Yan Wang

Qiang Zhou

Yichu Liu

Wan Li

Pingchao Li

Jinhong Zhu

Jiali Fang

Jingcai Hou

$\mathrm{Na} \mathrm{Li}$

Xingqiang Lai

Feifei Ma

Ning Li

Guanghui Li

Weiya Kong

Weiting Zhang

Jiale Kang

Jiali Li

Haoyu Chen

Liqiang Feng

Zheng Chen

Ling Chen

tianxing ji ( $\sim$ jitianxing7021@163.com )

Guangzhou Medical University Second Affiliated Hospital https://orcid.org/0000-0001-6760-9775

Research Article 
Keywords: Kidney transplant recipients, COVID-19, Humoral immunity, Cellular immunity, Inactivated vaccine

Posted Date: March 3rd, 2022

DOI: https://doi.org/10.21203/rs.3.rs-1406060/v1

License: (c) (1) This work is licensed under a Creative Commons Attribution 4.0 International License. Read Full License 


\section{Serum Unconjugated Bilirubin as a Predictor of Specific Cellular Immunity Response in Kidney Transplant Recipients after}

\section{Administration of SARS-CoV-2 Inactivated Vaccine}

Lei Zhang ${ }^{1 \#}$, Jiaqing Yang ${ }^{2 \#}$, Changchun Lai ${ }^{3 \#}$, Min Deng" ${ }^{4 \#}$, Xuanying Deng ${ }^{1}$, Yan Wang5, Qiang Zhou ${ }^{4}$, Yichu Liu ${ }^{6}$,

Li, Wan ${ }^{4}$, Pingchao $\mathrm{Li}^{6}$, Jinhong $\mathrm{Zhu}^{4}$, Jiali Fang ${ }^{1}$, Jingcai $\mathrm{Hou}^{7}, \mathrm{Na} \mathrm{Li}^{8}$, Xingqiang Lai ${ }^{1}$, Feifei $\mathrm{Ma}^{8}, \mathrm{Ning}^{4}{ }^{4}$, Guanghui $\mathrm{Li}^{1}$, Weiya Kong ${ }^{4}$, Weiting Zhang ${ }^{1}$, Jiale Kang ${ }^{4}$, Jiali Li ${ }^{1}$, Haoyu Chen ${ }^{4}$, Liqiang Feng ${ }^{6,10,11}$, Zheng Chen ${ }^{*}$, Ling Chen ${ }^{2,6,10,11^{*}}$, Tianxing $\mathrm{Ji}^{4^{*}}$

1. Kidney Transplant Department, The Second Affiliated Hospital of Guangzhou Medical University, 510260, P. R. China

2. State Key Laboratory of Respiratory Disease, Guangzhou Institute of Respiratory Health, First Affiliated Hospital of Guangzhou Medical University, Guangzhou, 510120, P. R. China

3. Clinical Laboratory Medicine Department, Maoming People's Hospital, Maoming, 525000, P. R. China

4. Clinical Laboratory Medicine Department, The Second Affiliated Hospital of Guangzhou Medical University, Guangzhou, 510260, P. R. China

5. Department of Pulmonary and Critical Care Medicine, Guangdong Second Provincial General Hospital, 510317, P. R. China

6. State Key Laboratory of Respiratory Disease, Guangzhou Institutes of Biomedicine and Health (GIBH), Chinese Academy of Sciences, Guangzhou, 510530, P. R. China

7. Organ transplant Department, Zhongshan city people's hospital, 528403, P. R. China

8. Critical Care Medicine Department, Integrated Hospital of Traditional Chinese Medicine of Southern Medical University, 510315, P. R. China

9. Obstetrical Department, Second Affiliated Hospital of Guangzhou Medical University, Guangzhou, 510260, P. R. China

10. State Key Laboratories of Respiratory Diseases, Guangdong-Hong Kong-Macao Joint Laboratory of Infectious Respiratory Disease, Guangzhou Institutes of Biomedicine and Health, Chinese Academy of Sciences, Guangzhou, China

11. Bioland Laboratory (GRMH-GDL), Guangzhou Institutes of Biomedicine and Health, Chinese Academy of Sciences, Guangzhou, 510530, P. R. China 
\# These authors contributed equally

\section{*Corresponding authors:}

1. Tianxing Ji, Clinical Laboratory Medicine Department, The Second Affiliated Hospital of Guangzhou Medical University, Guangzhou, 510260, P. R. China. Email: jitianxing7021@163.com,

2. Ling Chen, Bioland Laboratory (GRMH-GDL), Guangzhou Institutes of Biomedicine and Health, Chinese Academy of Sciences, Guangzhou, 510530, P. R. China; State Key Laboratory of Respiratory Disease, Guangzhou Institute of Respiratory Health, First Affiliated Hospital of Guangzhou Medical University, Guangzhou, 510120, P.

R. China. Email: chen_ling@gibh.ac.cn,

3. Zheng Chen, Kidney Transplant Department, the Second Affiliated Hospital of Guangzhou Medical University, 510260, P. R. China. Email: docchenzheng@163.com

\section{Word counts}

Abstract: 242

Main text: 2705

\section{Abstract \\ Purpose:}

The immunogenicity of SARS-CoV-2 vaccines is poor in kidney transplant recipients (KTRs). The factors related to poor immunogenicity to vaccination in KTRs are not well defined.

\section{Methods:}

An observational study was conducted in KTRs and healthy individuals who had received two doses of SARS-CoV-2 inactivated vaccine. IgG antibodies against the receptor-binding domain found in the S1 subunit of the spike protein, and against nucleocapsid protein were measured using enzyme-linked immunosorbent assay. Receptor-binding domain (RBD)-angiotensin-converting enzyme 2 interactionblocking antibodies were measured using commercial kits. T cell responses against the spike and nucleocapsid proteins were detected using enzyme-linked immunosorbent 
spot assay.

\section{Results:}

No severe adverse effects were observed in KTRs after first or second dose of SARSCoV-2 inactivated vaccine. IgG antibodies against the receptor- binding domain, and nucleocapsid protein were not effectively induced in a majority of KTRs after second dose of inactivated vaccine. Specific T cell immunity response was detectable in $32 \%$ $40 \%$ KTRs after second doses of inactivated vaccine. KTRs who developed specific T cell immunity were more likely to be female, and have lower levels of total bilirubin, unconjugated bilirubin, and blood tacrolimus concentration. Multivariate logistic regression analysis found that blood unconjugated bilirubin was significantly negatively associated with SARS-CoV-2 specific T cell immunity response in k KTRs.

\section{Conclusions:}

Specific T cell immunity response could be induced in 32\%-40\% KTRs after two doses of inactivated vaccine. Blood unconjugated bilirubin was negatively associated with specific cellular immunity response in KTRs following vaccination.

\section{Key words:}

Kidney transplant recipients, COVID-19, Humoral immunity, Cellular immunity, Inactivated vaccine. 


\section{Serum Unconjugated Bilirubin as a Predictor of Specific Cellular Immunity Response in Kidney Transplant Recipients after}

Administration of SARS-CoV-2 Inactivated Vaccine

\section{Introduction}

Numerous studies have shown that solid organ transplant recipients (SOTRs) requiring lifelong maintenance immunosuppression have a higher risk of SARS-CoV-2 infection and severe COVID-19.(1) Given that vaccination is considered as one of the best strategies for curbing the COVID-19 pandemic, several nephrology societies have called for kidney transplant recipients (KTRs) to be prioritized for the administration of SARS-CoV-2 vaccine.(2) The safety and efficacy of various SARS-CoV-2 vaccines in KTRs need to be urgently ascertained since these patients have been excluded from most clinical trials of SARS-CoV-2 vaccines.(3) A few clinical trials of mRNA vaccines have demonstrated that humoral and cellular immunity response was significantly lower in KTRs than in healthy participants due to the KTRs receiving life-long immunosuppression regimens. $(4,5)$ Further studies have demonstrated that three doses of mRNA vaccines could further enhance the antibody titers in SOTRs compared with two doses.(6) Recent data has demonstrated that inactivated vaccines could induce specific cellular immunity response but not humoral immunity response in some KTRs.(7) However, the risk factors associated with the poor response to COVID-19 vaccination in KTRs are not well-defined.(8)

\section{Methods}

\subsection{Subjects}

The study was conducted in accordance with the Declaration of Helsinki and was approved by the Ethics Committee of the Second Affiliated Hospital of Guangzhou Medical University (Approval No. 2021-hs-43). The clinical trial protocol was registered with the Chinese Clinical Trial Registry (No. ChiCTR2100049037, 
Registry's URL: https://www.chictr.org.cn/listbycreater.aspx). This observational study was conducted at the second affiliated hospital of Guangzhou medical university from June 20, 2021, to August 20, 2021. A total of 163 subjects were enrolled at transplant center after providing written informed consent. Of the 163 participants, 95 had received two doses of SARS-CoV-2 inactivated vaccine whereas 68 participants were unvaccinated. Of the 96 fully vaccinated participants, 43 were kidney transplant recipients (KTRs) whereas 52 were healthy. In the unvaccinated group, 38 were KTRs whereas 30 were healthy. None of the participants in the unvaccinated group had a history suggestive of symptomatic COVID infection. In the case of KTRs, the following data was extracted from the records: patient's clinical data including age, sex, medical history, medication history, kidney transplant time, body mass index, hematologic parameters (white blood cell counts, lymphocyte counts, platelet counts and hemoglobin), hepatic function (alanine aminotransferase, aspartate aminotransferase, and total bilirubin) and kidney function tests (serum creatinine, urine protein, and urine red cells). In the case of vaccinated individuals, the SARS-CoV-2 vaccine brand administered and adverse effects (AEs), if any, were noted.

\subsection{Sample processing}

Among the vaccinated participants, $10 \mathrm{~mL}$ blood was collected from 40 KTRs and 48 healthy participants (HPs) between $21 \pm 4$ days after the second dose of vaccine. Of whom, 17KTRs and 23HPs also participated in blood collection between $40 \pm 9$ days after second dose. Besides, blood was collected from another 3KTRs and 4HPs between $40 \pm 9$ days after second dose. In the unvaccinated group, $10 \mathrm{~mL}$ blood was drawn for determining the baseline value of SARS-CoV-specific humoral and cellular immunity. The plasma was separated by centrifugation (3000 rpm for $15 \mathrm{~min}$ ) and an equal amount of Roswell Park Memorial Institute (RPMI) 1640 culture medium (Gibco, USA) was added to the supernatant. This was then processed for anti-SARS-CoV-2 antibody detection. Peripheral blood mononuclear cells (PBMCs) were isolated from whole blood samples by Lymphoprep ${ }^{\mathrm{TM}}$ density gradient medium (Alere Tech, USA) for T cell immunity response analysis. 


\subsection{Anti-SARS-CoV-2 antibody detection}

IgG antibodies against receptor-binding domain (RBD), S1 domain of spike protein, (S1) and nucleocapsid proteins (NP) were detected using enzyme-linked immunosorbent assay (ELISA)(9). Briefly, RBD, S1 or NP was coated into the ELISA plate well. $100 \mu \mathrm{L}$ of diluted plasma (1:200) was then added into the well of the ELISA plate and incubated at $37^{\circ} \mathrm{C}$ for one hour. Then, $100 \mu \mathrm{L}$ of diluted anti-human $\operatorname{IgG}$ antibody (1:8000, Southern Biotech, USA) was added into the well of the ELISA plate and incubated at $37^{\circ} \mathrm{C}$ for one hour. Afterwards, $50 \mu \mathrm{L}$ of $3,3^{\prime}, 5,5^{\prime}-$ tetramethylbenzidine (TMB) solution (Neobioscience, China) was added and this mixture was further incubated at room temperature for 5-10 min. Finally, $50 \mu \mathrm{L}$ of $1 \mathrm{M}$ sulfuric acid $\left(\mathrm{H}_{2} \mathrm{SO}_{4}\right)$ solution was added to terminate the chromogenic reaction. The absorbance at $450 \mathrm{~nm}$ was obtained using a microplate absorbance reader (Tecan Sunrise, Switzerland).

\subsection{Surrogate SARS-CoV-2 neutralization test}

Anti-SARS-CoV-2 neutralizing antibody ELISA Kit (Vazyme Biotech, China) was used to qualitatively detect RBD-angiotensin-converting enzyme2 (ACE2) interaction blocking antibodies. Briefly, $80 \mu \mathrm{L}$ of horseradish peroxidase (HRP)-conjugated RBD solution was added into a 96-well dilution plate with $8 \mu \mathrm{L}$ plasma and $72 \mu \mathrm{L}$ sample dilution buffer, and incubated at $37^{\circ} \mathrm{C}$ for $30 \mathrm{~min}$. After that, $100 \mu \mathrm{L}$ of this plasma/HRP-conjugated RBD mixture was then transferred to a microplate coated with ACE2 and incubated at $37^{\circ} \mathrm{C}$ for $20 \mathrm{~min}$. Then $100 \mu \mathrm{L}$ TMB substrate solution was added after washing with diluted washing buffer, and incubated at room temperature for $15 \mathrm{~min}$. The reaction was stopped with $50 \mu \mathrm{L}$ of stop solution. Finally, the absorbance at $450 \mathrm{~nm}$ was obtained using a microplate absorbance reader (Tecan Sunrise, Switzerland).

\subsection{Detection of anti-SARS-CoV-2 T-cell frequencies}

SARS-CoV-2 spike, or NP-specific T lymphocytes were detected using interferon- $\gamma$ (IFN $\gamma$ ) enzyme-linked immunospot (EliSpot) assay. Fresh PBMCs were re-suspended 
in RPMI 1640 culture medium (Gibco, USA) supplemented with 10\% fetal bovine serum (Gibco, USA), 0.55 mM 2-hydroxyethylmercaptan (Gibco, USA), 2 mM Lglutamine (Gibco, USA), $1 \mathrm{mM}$ pyruvate (Gibco, USA), 1\% penicillin-streptomycin (Gibco, USA) and 10 mM N'-a-hydroxythylpiperazine-N'-ethanesulfanic acid (HEPES) (Gibco, USA). The concentration of PBMCs was determined using a hand-held automated cell counter (Millipore, USA). Then $2 \times 10^{5}$ PBMCs were added into each well of an anti-IFN $\gamma$ pre-coated EliSpot plate (Dakewe Biotech, China), and co-cultured with overlapping peptide pools of SARS-CoV-2 spike or NP for $24 \mathrm{~h}$ with dimethyl sulfoxide (Sigma, USA) as a negative control (NC). For positive control, $2 \times 10^{4} \mathrm{PBMCs}$ stimulated with staphylococcal enterotoxin B (1 $\mu \mathrm{g} / \mathrm{mL}$, Merck, Germany) were adopted. Diluted biotinylated antibody working solution $(100 \mu \mathrm{L})$ was added to the plate well and incubated at $37^{\circ} \mathrm{C}$ for $1 \mathrm{~h}$. After that, $100 \mu \mathrm{L}$ streptavidin-horseradish peroxidase working solution was added and incubated at $37^{\circ} \mathrm{C}$ for $1 \mathrm{~h}$. Afterwards, 100 $\mu \mathrm{L}$ 3-amino-9-ethylcarbazole (AEC) solution was added and incubated at room temperature for $30 \mathrm{~min}$. Finally, the spots were counted using the $\operatorname{ImmunoSpot}{ }^{\circledR} \mathrm{S} 6$ UV Analyzer (Cellular Technology Limited, USA). The means of duplicate wells were calculated and expressed as the number of spots per well.

\subsection{Statistical analysis}

Statistical analyses were performed using IBM SPSS Statistics 22.0 or GraphPad prism7.0. The Pearson chi-square was used to test differences in proportions. The t-test or Mann-Whitney U-test was utilized to explore the difference in continuous variables between two groups. One-way analysis of variance (ANOVA) tests were applied for comparing the means of continuous variables in the four groups. Paired data was analyzed using paired t-tests. The correlation between anti-SARS-CoV-2 antibody and spike or NP-specific T cell frequency was determined using the Pearson correlation coefficient. A two-sided p-value $<0.05$ was considered statistically significant.

\section{Results}

\subsection{Baseline characteristics of the study subjects}


The baseline characteristics of participants included in this study are summarized in Table 1. A majority of KTRs in the study were male (unvaccinated KTRs 22/30, vaccinated KTRs 35/43). The number of males was significantly higher in KTRs as compared with that in HPs (unvaccinated HPs 17/38, vaccinated healthy participants $28 / 58, p<0.0001)$. The mean time after transplantation in vaccinated KTRs was $68.24 \pm 51.41$ months, and significantly longer than that in the unvaccinated KTRs (27.37 \pm 21.17 months, $p=0.000)$. None of the participants had a history suggestive of symptomatic COVID-19.

\subsection{Adverse effects}

The vaccine brands received for the first and second doses in participants and the adverse effects seen are summarized in Table 2. The vaccine brand used for the second dose in KTRs and HPs was significantly different ( $p=0.001$ ), with 90.4\% HPs and 62.8\% KTRs having received CoronaVac. Adverse reactions to vaccine were mild, transient, and self-limiting.

\subsection{Humoral immunity response of KTR after vaccination}

Antibody response to SARS-CoV-2 was assessed in all individuals 14-28 days and 2956 days after the second dose of inactivated vaccine. Anti-S1 antibody IgG was effectively induced in most HPs after two doses of inactivated vaccine (Figure1A), with $77.1 \%$ (37/48) being positive between $21 \pm 4$ days after the second dose, and $51.9 \%$ (14/27) being positive in $40 \pm 9$ days after the second dose (Table3). The blood anti-S1 antibody IgG level in KTRs was significantly lower than in HPs ( $p<0.0001)$ (Figure1A), with $7.5 \%$ (3/40) of KTRs having anti-S1 antibody IgG positivity in $21 \pm 4$ days after the

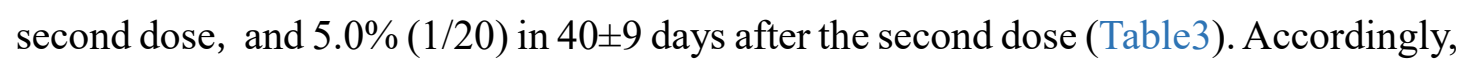
seroconversion for anti-RBD IgG antibody was observed in most of the HPs but only in two KTRs after the second dose of inactivated vaccine (Figure2B and Table3). RBDACE2 interaction-blocking assay was performed to further determine the virus neutralizing capacity of HPs and KTRs after the second dose of inactivated vaccine. The results suggest that $93.8 \%$ (45/48) HPs developed virus-neutralizing antibody after two doses of inactivated vaccine, however, only 5\% (2/40) KTRs developed virus- 
neutralizing antibody after two doses of inactivated vaccine (Figure1C and Table3). Similarly anti-NP-antibody IgG was increased in most of the HPs after two doses of inactivated vaccine (Figure1D and Table3). However, almost all the KTRs had a blunted seroconversion of anti-NP-antibody IgG after two doses of inactivated vaccine (Figure1D and Table3). Statistical analysis was carried out to study the effect of gender and vaccine brand as factors for seroconversion between KTRs and HPS. The results show that the anti-spike IgG antibody positivity rate (PR) in females was significantly higher than that in males (Supplemental Table1). Anti-NP IgG PR in HPs receiving the second dose of the vaccine brand Sinopharm BIBP was higher than those who had received CoronaVac as the second dose. (Supplemental Table2). In summary, there was good antibody response in immunocompetent individuals after two doses of inactivated vaccine, but this was not the case with KTRs.

\section{4 $T$ cells immunity response of KTR after vaccination}

To determine whether the cellular immunity could be induced in KTRs, the ELISPOT was performed to evaluate the cellular immunity against the two major structural proteins of SARS-CoV-2, spike, and NP. As shown in Figure2, T cells reactive to spike or NP were significantly increased in HPs after the second dose of inactivated vaccine compared with unvaccinated HPs. Paired analysis demonstrated spike-specific T cell frequency in HPs increased furtherly in $40 \pm 9$ days after the second dose of inactivated vaccine compared with that seen in $21 \pm 4$ days after the second dose, but this was not observed with NP-specific T cells (Figure2B and D). An increase in spike or NPspecific T cell frequency was also observed in KTRs, $21 \pm 4$ days after the second dose of vaccine though this response was lower than that observed in HPs (Figure2A and C). This difference was statistically significant (Table4). Paired analysis indicated spikespecific $\mathrm{T}$ cell frequency in KTRs further increased marginally in $40 \pm 9$ days after the second dose of inactivated vaccine as compared with that at $21 \pm 4$ days after the second dose, but this was not observed for NP-specific T cells (Figure2B and D). Both spike and NP-specific T cell frequency in KTRs at $21 \pm 4$ days after the second dose of vaccine was comparable with that observed in HPs (Figure2A and C). The positivity rate of 
spike-specific T cell immunity response in KTRs at $40 \pm 9$ days after the second dose was not different from that in HPs (40.0\% versus $66.7 \%, p=0.069)$ (Table4). However, the positivity rate of NP-specific T cell immunity response in KTRs between $40 \pm 9$ days after second dose was significantly lower than that in HPs $(35.0 \%$ versus $70.4 \%$, $p=0.016$ ). Furthermore, the spike and NP-specific T cell frequency in HPs was significantly related to the anti-SARS-CoV-2 antibody response including antiRBD/anti-S1/anti-NP IgG, and neutralizing antibodies (Figure S1A-I). However, this correlation was not found in KTRs (Figure S1G-P), indicating a dichotomous humoral and cellular immunity response in the KTRs after vaccination. These results indicate that SARS-CoV-2-specific cellular immunity could be induced in some KTRs after administration of inactivated vaccine.

\subsection{Factors associated with lower SARS-CoV-2 specific $T$ cell immunity response in KTRs}

We also explored factors associated with SARS-CoV-2 specific T cell immunity response in KTRs, including age, sex, body mass index, hematologic parameters (white blood cell counts, lymphocyte counts, platelet counts and hemoglobin), hepatic function and kidney function tests, immunosuppression medicine used, induction agent used and comorbidities. The results demonstrated that KTRs with SARS-CoV-2 specific T cells immunity had a higher frequency of females $(p=0.023)$, and lower total bilirubin $(p=0.017)$, indirect bilirubin $(p=0.009)$ and blood tacrolimus concentration $(p=0.004)$ compared with KTRs without SARS-CoV-2 specific T cell immunity response after two doses of inactivated vaccine (Table5). Multivariate logistic regression analysis was performed using independent variables with $p<0.2$ in the univariate analysis (Table5). This demonstrated that blood unconjugated bilirubin levels were significantly negatively associated with SARS-CoV-2 specific T cells immunity response in KTRs $(\mathrm{OR}=0.723 ; 95 \%$ CI $0.537-0.972, p=0.032)$.

\section{Discussion}

Information regarding the safety and efficacy of vaccines in SOTRs is urgently needed.(1, 10) Currently, mRNA vaccines and replication-defective viral-vectored 
vaccines are approved for use in immunocompromised patients (11). Our study as well as other studies have shown that in KTRs, AEs with inactivated vaccine were mild, and comparable with those in healthy individuals, implying that inactivated vaccine is safe in immunocompromised patients (7)

The efficacy of vaccination in immunocompromised patients has been a matter of concern. Studies have shown that vaccination of KTRs with mRNA vaccine resulted in lower humoral and cellular immunity response after two doses as compared with healthy individuals. ${ }^{(4)}$ It has been found that a lower humoral immunity response is observed in KTRs after two doses of inactivated vaccine as well, placing them at high risk of breakthrough infections. $(12,13)$ Therefore, it is possible that a third dose of SARS-CoV-2 vaccine may be needed in these patients. $(6,14)$

The T cell response to two doses of inactivated COVID vaccines in KTRs has been found to be better than the humoral response.(15) In addition, the virus with novel mutations of the spike proteins does not easily escape T-cell-mediated immunity elicited by the wild type spike protein.(15) The role of $\mathrm{T}$ cell immunity response has been found to be critical in virus clearance in COVID-19 patients with low levels of antibody, such as patients with hematologic cancer.(16) As shown in an earlier study(7), our results also show that KTRs had enhanced NP and spike-specific T cell response after the second dose of inactivated vaccine. This enhanced NP and spike-specific T cell immunity response could potentially provide synergistic antiviral effects and prevent severe COVID-19 following SARS-CoV-2 infection. ${ }^{(17,18)}$ A recent study has demonstrated that replication-deficient adenoviral vector vaccines $\mathrm{ChAdOx} 1$ could be more effective in inducing $T$ cell response as compared with mRNA vaccines.(19) Thus, a third dose with an adenoviral vector vaccine might be a better strategy for improving T cell immunity response. $(6,14)$

In our study we found that specific $\mathrm{T}$ cell immunity response to SARS-CoV-2 inactivated vaccine in KTRs was negatively associated with blood unconjugated bilirubin, which is a test for hepatic function.(20) Apart from indicating liver dysfunction, unconjugated bilirubin in physiological ranges can function as an 
immunosuppressant, by impairment of antigen presentation in macrophages and inhibition of CD4+ T cell responses, especially Th1 response (IL-2 and IFN- $\gamma$ ). These mechanisms may explain the lower protective T cell immunity response to SARS-CoV2 in KTRs following vaccination in those with elevated unconjugated bilirubin.(21-23) As shown in previous studies, we also found that the $\mathrm{T}$ cell immunity response in KTRs after two doses of inactivated vaccine was negatively related to the blood tacrolimus concentration though this was not statistically significant. ${ }^{(19)}$ It should be noted that the majority of our patients were treated with mycophenolate mofetil (MMF), which may have contributed to impaired humoral response following vaccination.(24) Whether the low humoral immunity is related to the dysregulation of the T cell response by MMF needs to be further investigated.

In summary, this study demonstrates that SARS-CoV-2 specific cellular immunity response could be effectively induced in some KTRs after administration of two doses of inactivated vaccine. Blood unconjugated bilirubin levels were negatively associated with SARS-CoV-2 specific cellular immunity response in KTRs. Further prospective studies with an adequate sample size are needed to determine the role of unconjugated bilirubin levels in predicting the cellular response to inactivated vaccines in KTRs.

\section{Funding}

This study received the following funding: Guangzhou Health Science and Technology Project (20201A011078), Guangzhou Science and Technology Project (201904010214, 202102010094), Guangdong Basic and Applied Basic Research Foundation (2021A1515012550), National Natural Science Foundation of China (82061138006), Key clinical specialty of Guangzhou Medical University (0F03031) and Major clinical science and technology projects in Guangzhou (2019ZD12).

\section{Conflicts of interests}

All authors declare no competing interests. 


\section{Authors' contributions}

Conceptualization: Ji TX, Clinical sample and data collection: Zhang L, Lai CC, Wang Y, Deng XY, Zhou Q, Zhu JH, Hou JC, Fang JL, Li N, Lai XQ, Ma FF, Li N, Li GH, Kong YW, Zhang WT, Kang JL, Li JL, Chen HY, and Chen HY. Methodology devising and performing experiments and Data analysis: Yang JQ, Zhang L, Deng M, Ji TX, Liu YC, Feng LQ and Li PC. Writing original draft: Ji TX, Zhang L, and Yang JQ. Funding acquisition: Ji TX, Chen L, Chen Z, Zhang L, and Zhou Q. Supervision: Ji TX, Chen L, and Chen Z. All authors contributed to data analysis and interpretation and edited the manuscript.

\section{Acknowledgment}

We thank all participants in this study. We would like to thank TopEdit (www.topeditsci.com) for its linguistic assistance during the preparation of this manuscript.

\section{Availability of data and material}

Not applicable

\section{Ethics Approval}

This study was performed in line with the Declaration of Helsinki and was approved by the Ethics Committee of the Second Affiliated Hospital of Guangzhou Medical University (Approval No. 2021-hs-43). The clinical trial protocol was registered with the Chinese Clinical Trial Registry (No. ChiCTR2100049037, Registry's URL: https://www.chictr.org.cn/listbycreater.aspx)

\section{Consent to Participate}

Informed consent was obtained from all individual participants enrolled in the study. 


\section{Consent for Publication}

The authors affirm that all enrolled participants provided informed consent for publication of the data.

\section{Reference}

1. Akalin E, Azzi Y, Bartash R, Seethamraju H, Parides M, Hemmige V, et al. Covid-19 and Kidney Transplantation. The New England journal of medicine. 2020;382(25):2475-7.

2. Kronbichler A, Anders HJ, Fernandez-Juarez GM, Floege J, Goumenos D, Segelmark M, et al. Recommendations for the use of COVID-19 vaccines in patients with immune-mediated kidney diseases. Nephrology, dialysis, transplantation : official publication of the European Dialysis and Transplant Association - European Renal Association. 2021.

3. Glenn DA, Hegde A, Kotzen E, Walter EB, Kshirsagar AV, Falk R, et al. Systematic Review of Safety and Efficacy of COVID-19 Vaccines in Patients With Kidney Disease. Kidney international reports. 2021;6(5):1407-10.

4. Sattler A, Schrezenmeier E, Weber UA, Potekhin A, Bachmann F, Straub-Hohenbleicher H, et al. Impaired humoral and cellular immunity after SARS-CoV2 BNT162b2 (Tozinameran) primeboost vaccination in kidney transplant recipients. The Journal of clinical investigation. 2021;131(14):e150175.

5. Boyarsky BJ, Werbel WA, Avery RK, Tobian AAR, Massie AB, Segev DL, et al. Antibody Response to 2-Dose SARS-CoV-2 mRNA Vaccine Series in Solid Organ Transplant Recipients. Jama. 2021;325(21):2204-6.

6. Kamar N, Abravanel F, Marion O, Couat C, lzopet J, Del Bello A. Three Doses of an mRNA Covid-19 Vaccine in Solid-Organ Transplant Recipients. The New England journal of medicine. 2021;385(7):661-2.

7. Bruminhent J, Setthaudom C, Chaumdee P, Boongird S, Kiertiburanakul S, Malathum K, et al. SARS-CoV-2-specific Humoral and Cell-mediated Immune Responses after Immunization with Inactivated COVID-19 Vaccine in Kidney Transplant Recipients (CVIM 1 Study). American journal of transplantation : official journal of the American Society of Transplantation and the American Society of Transplant Surgeons. 2021.

8. Azzi Y, Raees H, Wang T, Cleare L, Liriano-Ward L, Loarte-Campos P, et al. Risk factors associated with poor response to COVID-19 vaccination in kidney transplant recipients. Kidney international. 2021;100(5):1127-8.

9. Lai C, Liu X, Yan Q, Lv H, Zhou L, Hu L, et al. Low Innate Immunity and Lagged Adaptive Immune Response in the Re-Tested Viral RNA Positivity of a COVID-19 Patient. Frontiers in immunology. 2021;12:664619.

10. Kronbichler A, Gauckler P, WindpessI M, II Shin J, Jha V, Rovin BH, et al. COVID-19: implications for immunosuppression in kidney disease and transplantation. Nat Rev Nephrol. 2020;16(7):365-7. 
11. WindpessI M, Bruchfeld A, Anders HJ, Kramer H, Waldman M, Renia L, et al. COVID-19 vaccines and kidney disease. Nat Rev Nephrol. 2021;17(5):291-3.

12. Brosh-Nissimov T, Orenbuch-Harroch E, Chowers M, Elbaz M, Nesher L, Stein M, et al. BNT162b2 vaccine breakthrough: clinical characteristics of 152 fully vaccinated hospitalized COVID-19 patients in Israel. Clinical microbiology and infection : the official publication of the European Society of Clinical Microbiology and Infectious Diseases. 2021;S1198-743X(21):003670 .

13. Bergwerk M, Gonen T, Lustig Y, Amit S, Lipsitch M, Cohen C, et al. Covid-19 Breakthrough Infections in Vaccinated Health Care Workers. The New England journal of medicine. 2021.

14. Ducloux D, Colladant M, Chabannes M, Yannaraki M, Courivaud C. Humoral response after three doses of BNT162b2 mRNA COVID-19 vaccine in patients on hemodialysis. Kidney international. 2021;100(3):702-4.

15. Cromer D, Juno JA, Khoury D, Reynaldi A, Wheatley AK, Kent SJ, et al. Prospects for durable immune control of SARS-CoV-2 and prevention of reinfection. Nature reviews Immunology. 2021;21(6):395-404.

16. Bange EM, Han NA, Wileyto P, Kim JY, Gouma S, Robinson J, et al. CD8(+) T cells contribute to survival in patients with COVID-19 and hematologic cancer. Nature medicine. 2021;27(7):1280 9 .

17. Dangi T, Class J, Palacio N, Richner JM, Penaloza MacMaster P. Combining spike- and nucleocapsid-based vaccines improves distal control of SARS-CoV-2. Cell reports. 2021;36(10):109664.

18. Che Y, Liu X, Pu Y, Zhou M, Zhao Z, Jiang R, et al. Randomized, double-blinded and placebocontrolled phase II trial of an inactivated SARS-CoV-2 vaccine in healthy adults. Clinical infectious diseases : an official publication of the Infectious Diseases Society of America. 2020:ciaa1703.

19. Prendecki M, Clarke C, Edwards H, McIntyre S, Mortimer P, Gleeson S, et al. Humoral and Tcell responses to SARS-CoV-2 vaccination in patients receiving immunosuppression. Annals of the rheumatic diseases. 2021.

20. Parker R, Cabezas J, Altamirano J, Arab JP, Ventura-Cots M, Sinha A, et al. Trajectory of Serum Bilirubin Predicts Spontaneous Recovery in a Real-World Cohort of Patients With Alcoholic Hepatitis. Clinical gastroenterology and hepatology : the official clinical practice journal of the American Gastroenterological Association. 2021.

21. Liu Y, Li P, Lu J, Xiong W, Oger J, Tetzlaff W, et al. Bilirubin possesses powerful immunomodulatory activity and suppresses experimental autoimmune encephalomyelitis. Journal of immunology. 2008;181(3):1887-97.

22. Tsai MT, Tarng DC. Beyond a Measure of Liver Function-Bilirubin Acts as a Potential Cardiovascular Protector in Chronic Kidney Disease Patients. International journal of molecular sciences. 2018;20(1).

23. Sundararaghavan VL, Binepal $S$, Stec DE, Sindhwani $P$, Hinds TD, Jr. Bilirubin, a new therapeutic for kidney transplant? Transplantation reviews. 2018;32(4):234-40.

24. Kantauskaite M, Muller L, Kolb T, Fischer S, Hillebrandt J, Ivens $K$, et al. Intensity of mycophenolate mofetil treatment is associated with an impaired immune response to SARS-CoV2 vaccination in kidney transplant recipients. American journal of transplantation : official journal of the American Society of Transplantation and the American Society of Transplant Surgeons. 2021. 


\section{Tables}

\section{Table1. Patient's characteristics}

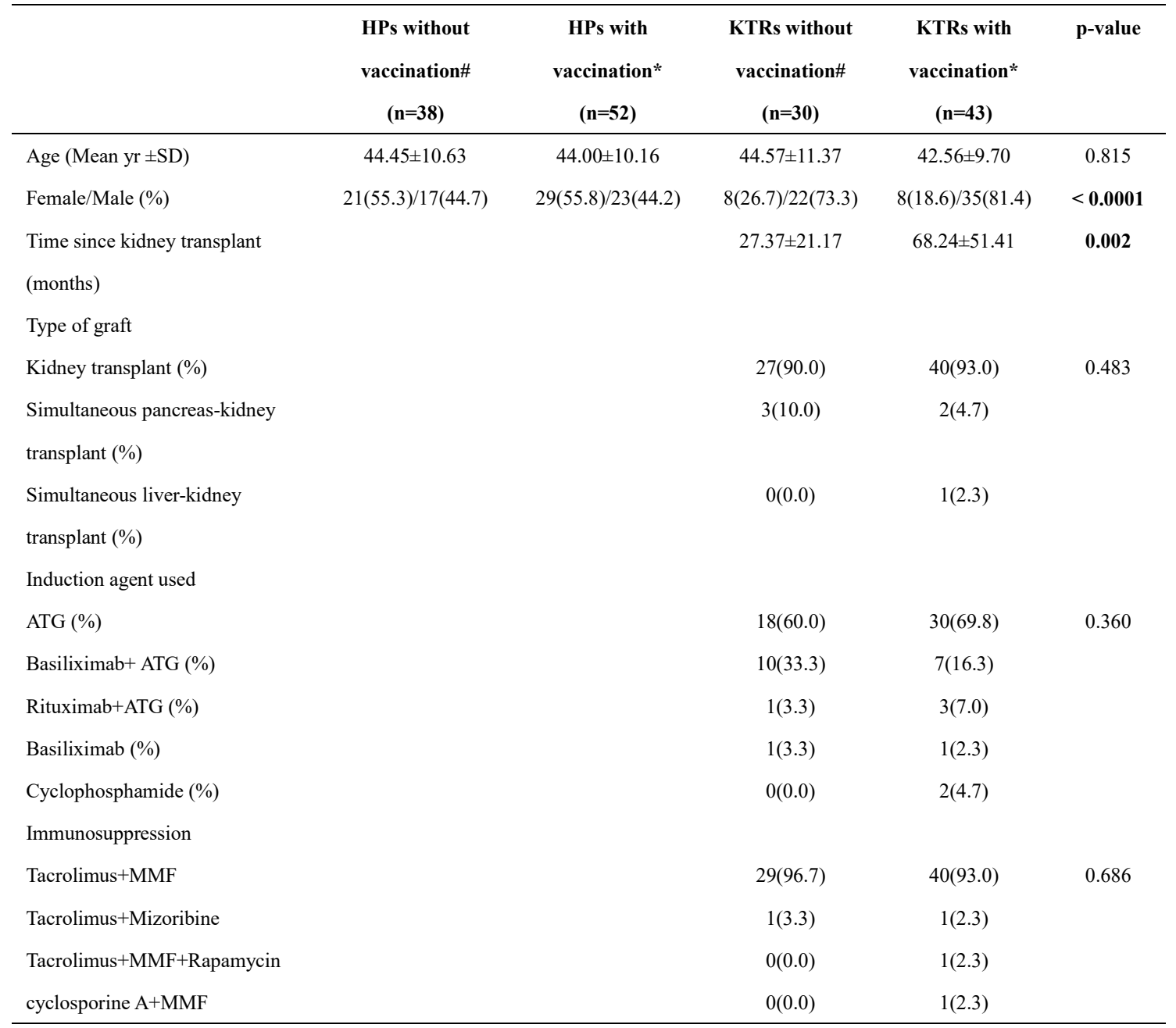

KTRs: kidney transplants recipients, HPs: healthy participants, * kidney transplants recipients and healthy participants with administration of inactivated vaccine; \#kidney transplants recipients and healthy participants without vaccination of SARS-CoV-2 inactivated vaccine; MMF: mycophenolate mofetil; ATG: anti-thymocyte globulin. 
Table2. The adverse effects of kidney transplants recipients and healthy participants after first and second dose of inactivated vaccine.

\begin{tabular}{|c|c|c|c|}
\hline & HPs $(n=52)$ & KTRs $(n=43)$ & p-value \\
\hline \multicolumn{4}{|c|}{ Inactivated Vaccine brand of the first dose } \\
\hline Sinopharm BIBP (\%) & $21(40.4)$ & $16(37.2)$ & 0.752 \\
\hline CoronaVac $(\%)$ & $31(59.6)$ & $27(62.8)$ & \\
\hline \multicolumn{4}{|c|}{ Inactivated Vaccine brand of the second dose* } \\
\hline Sinopharm BIBP (\%) & $5(9.6)$ & $16(37.2)$ & 0.001 \\
\hline CoronaVac $(\%)$ & $47(90.4)$ & $27(62.8)$ & \\
\hline \multicolumn{4}{|c|}{ Time interval between first dose and second dose (day)* } \\
\hline & $26.69 \pm 9.02$ & $26.56 \pm 8.15$ & 0.940 \\
\hline \multicolumn{4}{|c|}{ Adverse effects after first dose } \\
\hline No abnormalities (\%) & $32(61.5)$ & $27(62.8)$ & 0.844 \\
\hline Pain at injection site (\%) & $11(21.1)$ & $11(25.6)$ & \\
\hline Fatigue (\%) & $7(13.5)$ & $4(9.3)$ & \\
\hline Dizzy (\%) & $1(1.9)$ & $1(2.3)$ & \\
\hline Allergy (\%) & $1(1.9)$ & $0(0.0)$ & \\
\hline \multicolumn{4}{|c|}{ Adverse effects after second dose* } \\
\hline No abnormalities (\%) & $37(71.2)$ & $28(67.4)$ & 0.569 \\
\hline Pain at injection site (\%) & $10(19.2)$ & $7(16.3)$ & \\
\hline Fatigue (\%) & $4(7.7)$ & $5(11.6)$ & \\
\hline Dizziness (\%) & $0(0.0)$ & $1(2.3)$ & \\
\hline Diarrhea (\%) & $0(0.0)$ & $1(2.3)$ & \\
\hline Runny nose (\%) & $1(1.9)$ & $0(0.0)$ & \\
\hline
\end{tabular}

KTRs: kidney transplants recipients, HPs: healthy participants. 
Table3. The spike or nucleocapsid protein specific $\operatorname{IgG}$ antibody positive rate in kidney transplants recipients and healthy participants after second dose of inactivated vaccine.

\begin{tabular}{|c|c|c|c|}
\hline & KTRs & HCs & p-value \\
\hline $21 \pm 4$ days after $2^{\text {nd }}$ dose & $(n=40)$ & $(n=48)$ & \\
\hline Anti-S1 IgG antibody positive (\%) & $3(7.5)$ & $37(77.1)$ & $<0.0001$ \\
\hline Anti-RBD IgG antibody positive (\%) & $2(5.0)$ & $32(66.7)$ & $<0.0001$ \\
\hline Neutralizing antibody positive (\%) & $2(5.0)$ & $45(93.8)$ & $<0.0001$ \\
\hline Anti-NP IgG antibody positive (\%) & $1(2.5)$ & $26(54.2)$ & $<0.0001$ \\
\hline $40 \pm 9$ days after $2^{\text {nd }}$ dose & $(\mathbf{n}=\mathbf{2 0})$ & $(n=27)$ & \\
\hline Anti-S1 IgG antibody positive (\%) & $1(5.0)$ & $14(51.9)$ & 0.001 \\
\hline Anti-RBD IgG antibody positive (\%) & $1(5.0)$ & $12(44.4)$ & 0.003 \\
\hline Neutralizing antibody positive (\%) & $2(10.0)$ & $24(88.9)$ & $<0.0001$ \\
\hline Anti-NP IgG antibody positive (\%) & $0(0.0)$ & $11(40.7)$ & 0.001 \\
\hline
\end{tabular}

KTRs: kidney transplants recipients, HPs: healthy participants, S1: The S1 domain of the spike protein. RBD: receptor binding domain, NP: nucleocapsid protein 
Table4. The spike or nucleocapsid protein specific $\mathrm{T}$ cell positive rate in kidney transplants recipients and healthy participants after second dose of inactivated vaccine.

\begin{tabular}{llll}
\hline & KTRs & HPs & p-value \\
\hline $\mathbf{2 1} \pm \mathbf{4}$ days after 2 $\mathbf{2}^{\text {nd }}$ dose & $(\mathrm{n}=40)$ & $(\mathrm{n}=48)$ & \\
Spike specific T cell positive (\%) & $11(25.6)$ & $26(54.2)$ & $\mathbf{0 . 0 1 2}$ \\
NP specific T cell positive (\%) & $14(33.3)$ & $28(58.3)$ & $\mathbf{0 . 0 2 9}$ \\
Spike and NP specific T cell positive (\%) & $13(32.5)$ & $31(64.6)$ & $\mathbf{0 . 0 0 3}$ \\
$\mathbf{4 0 \pm 9 d a y s ~ a f t e r ~ 2 ~} \mathbf{2}^{\text {nd }}$ dose & $(\mathrm{n}=20)$ & $(\mathrm{n}=27)$ & \\
Spike specific T cell positive (\%) & $8(40.0)$ & $18(66.7)$ & 0.069 \\
NP specific T cell positive (\%) & $7(35.0)$ & $19(70.4)$ & $\mathbf{0 . 0 1 6}$ \\
Spike and NP specific T cell positive (\%) & $8(40.0)$ & $20(41.7)$ & $\mathbf{0 . 0 1 9}$ \\
\hline
\end{tabular}

KTRs: kidney transplants recipients, HPs: healthy participants, S1: The S1 domain of the spike protein. RBD: receptor binding domain, NP: nucleocapsid protein 
Table5. Comparative analysis of baseline characteristics of kidney transplant recipients with and without SARS-CoV-2 specific T cell immunity response after two doses of inactivated vaccine.

\begin{tabular}{|c|c|c|c|}
\hline & $\begin{array}{c}\text { KTRs without SARS-CoV-2 } \\
\text { specific } T \text { cells immunity(n=26) }\end{array}$ & $\begin{array}{c}\text { KTRs with SARS-CoV-2 } \\
\text { specific T cells immunity }(n=17)\end{array}$ & $p$-value \\
\hline Female sex & $2(7.7)$ & $6(35.3)$ & 0.023 \\
\hline Age(year) & $44.5(15)$ & $45.5(20)$ & 0.517 \\
\hline Body mass index & $23.2(3.9)$ & $21.6(4.45)$ & 0.243 \\
\hline White blood cell $(\times 109)$ & $6.51(2.8)$ & $7.91(3.91)$ & 0.501 \\
\hline Lymphocyte $(\times 109)$ & $1.58(0.99)$ & $1.98(0.91)$ & 0.115 \\
\hline Platelet $(\times 109)$ & 198(76) & 195(97) & 0.224 \\
\hline Hemoglobin $(g / L)$ & $145(21)$ & $139(32)$ & 0.196 \\
\hline Creatinine (umol/L) & $115.3(26)$ & $101.8(42)$ & 0.168 \\
\hline Alanine aminotransferase $(\mathrm{U} / \mathrm{L})$ & $20.4(9)$ & $16.25(15)$ & 0.412 \\
\hline Aspartate aminotransferase (U/L) & 20.3(4.95) & $20.1(4.75)$ & 0.827 \\
\hline Total bilirubin (umol/L) & $15.0(9.60)$ & $9.6(4.00)$ & 0.017 \\
\hline Ungonjugated bilirubin(umol/L) & $11.9(8.5)$ & 7.8(3.68) & 0.009 \\
\hline Conjugated bilirubin(umol/L) & $3.0(2.40)$ & $2.20(1.33)$ & 0.224 \\
\hline Urine protein positive & $5(19.2)$ & $1(5.9)$ & 0.217 \\
\hline Urine red cells positive & $8(30.8)$ & $5(29.4)$ & 0.925 \\
\hline Blood tacrolimus concentration (ng/mL) & $6.25(1.75)$ & $5.25(1.02)$ & 0.004 \\
\hline $\begin{array}{l}\text { Interval between vaccination and } \\
\text { transplant(months) }\end{array}$ & $46.5(40)$ & $70.5(70)$ & 0.117 \\
\hline \multicolumn{4}{|l|}{ Immunosuppression } \\
\hline Tacrolimus + MMF + Prednisone & $24(92.4)$ & $16(94.1)$ & 0.414 \\
\hline $\mathrm{CsA}+\mathrm{MMF}+$ Prednisone & $1(3.8)$ & $0(0)$ & \\
\hline Tacrolimus + Mizoribine + Prednisone & $0(0)$ & 1(5.9) & \\
\hline Tacrolimus + MMF + Rapa + Prednisone & $1(3.8)$ & $0(0)$ & \\
\hline \multicolumn{4}{|l|}{ Induction agent used } \\
\hline ATG & $16(61.5)$ & $14(82.4)$ & 0.193 \\
\hline Basiliximab+ATG & $6(23)$ & $1(5.9)$ & \\
\hline Rituximab + ATG & $3(11.5)$ & $0(0)$ & \\
\hline Basiliximab & $0(0)$ & $1(5.9)$ & \\
\hline CTX & $1(3.8)$ & $1(5.9)$ & \\
\hline \multicolumn{4}{|l|}{ Type of graft } \\
\hline Kidney transplant (\%) & $24(92.3)$ & $16(94.1)$ & 0.242 \\
\hline Simultaneous pancreas-kidney transplant (\%) & $2(7.7)$ & $0(0)$ & \\
\hline Simultaneous liver-kidney transplant (\%) & $0(0)$ & $1(5.9)$ & \\
\hline \multicolumn{4}{|l|}{ Comorbidity } \\
\hline Coronary disease & $2(7.7)$ & $0(0)$ & 0.242 \\
\hline Urinary infection & $1(3.8)$ & $1(5.9)$ & 0.757 \\
\hline Diabetes & $3(11.5)$ & $2(11.8)$ & 0.982 \\
\hline
\end{tabular}


Supplemental table1. Comparison of humoral and cellular immunity response between female and male in healthy participants $21 \pm 4$ days after second dose of inactivated vaccine.

\begin{tabular}{llll}
\hline & Female & Male & p-value \\
\hline $\mathbf{4 0} \pm \mathbf{9}$ days after $\mathbf{2}^{\text {nd }}$ dose & $\mathbf{( n = 2 7 )}$ & $\mathbf{( n = 2 1 )}$ & \\
Anti-S1 IgG antibody positive/negative (\%) & $23(85.2)$ & $14(66.7)$ & 0.130 \\
Anti-RBD IgG antibody positive/negative (\%) & $21(77.8)$ & $11(52.4)$ & 0.064 \\
RBD-ACE2 blocking antibody positive (\%) & $27(100)$ & $18(85.7)$ & $\mathbf{0 . 0 4 3}$ \\
Anti-NP IgG antibody positive/negative (\%) & $14(51.9)$ & $12(57.1)$ & 0.715 \\
Spike specific T cell positive/negative (\%) & $18(66.7)$ & $8(38.1)$ & $\mathbf{0 . 0 4 9}$ \\
NP specific T cell positive/negative (\%) & $18(66.7)$ & $10(47.6)$ & 0.184 \\
\hline
\end{tabular}

S1: The S1 domain of the spike protein. RBD:receptor binding domain, ACE2: angiotensin-converting enzyme 2, NP: nucleocapsid protein 
Supplemental table2. Comparison of humoral and cellular immunity response of second dose with Coronavac and Sinopharm BIBP in healthy participants $21 \pm 4$ days after first or second dose of inactivated vaccine.

\begin{tabular}{llll}
\hline & Coronavac & Sinopharm BIBP & $\boldsymbol{p}$-value \\
\hline 40 \pm 9days after 2nd dose & $\mathbf{( n = 4 3 )}$ & $\mathbf{( n = 5 )}$ & \\
Anti-S1 IgG antibody positive (\%) & $33(76.7)$ & $4(80.0)$ & 0.870 \\
Anti-RBD IgG antibody positive (\%) & $29(67.44)$ & $3(60.0)$ & 0.738 \\
RBD-ACE2 blocking antibody positive (\%) & $41(95.3)$ & $4(80.0)$ & 0.180 \\
Anti-NP IgG antibody positive (\%) & $21(48.8)$ & $5(100.0)$ & $\mathbf{0 . 0 3 0}$ \\
Spike specific T cell positive (\%) & $22(51.1)$ & $4(80.0)$ & 0.221 \\
NP specific T cell positive (\%) & $24(55.8)$ & $4(80.0)$ & 0.299 \\
\hline
\end{tabular}

S1: The S1 domain of the spike protein. RBD:receptor binding domain, ACE2: angiotensin-converting enzyme 2, NP: nucleocapsid protein 


\section{Figure legends}

Figure1. Anti-SARS-CoV-2 antibodies in kidney transplant recipients (KTR) and healthy participants (HPs) at $21 \pm 4$ days (KTRs1 and HPs1) and 40 \pm 9 days (KTRs2 and HPs2) after the second dose of SARS-CoV-2 inactivated vaccine compared with KTRs or HPs without vaccination (KTRs0 and HPs0). (A) The optical density of anti-S1 IgG antibody in KTRs and HPs without vaccination and in $21 \pm 4$ days and $40 \pm 9$ days after the second dose of SARSCoV-2 inactivated vaccine. (B) The optical density of anti-receptor binding domain (RBD) IgG antibody in KTRs and HPs without vaccination and in $21 \pm 4$ days and $40 \pm 9$ days after the second dose of SARS-CoV-2 inactivated vaccine. (C) The RBD-Angiotensin I Converting Enzyme 2 (ACE2) interaction blocking antibody in KTRs and HPs without vaccination and in $21 \pm 4$ days and $40 \pm 9$ days after the second dose of SARS-CoV-2 inactivated vaccine. (D) The optical density of anti-receptor binding domain (RBD) IgG antibody in KTRs and HPs without vaccination and in $21 \pm 4$ days and $40 \pm 9$ days after the second dose of SARS-CoV-2 inactivated vaccine. The horizontal dotted line indicates the cut-off value for positivity. The cut-off value was calculated using mean \pm 3 standard deviation (SD) optical density of plasma samples from HPs and KTRs without vaccination.

Figure2. T cell responses to pooled peptides from SARS-CoV-2 spike and nucleocapsid protein (NP) in kidney transplant recipients (KTR) and healthy participants (HPs) at $21 \pm 4$ days (KTRs1 and HPs1) and $40 \pm 9$ days (KTRs2 and HPs2) after the second dose of SARS-CoV-2 inactivated vaccine compared with KTRs or HPs without vaccination (KTRs0 and HPs0). (A) T cell responses targeted against SARS-CoV-2 spike in KTRs and HPs without vaccination, or at $21 \pm 4$ days and $40 \pm 9$ days after the second dose of inactivated vaccine. (B) The kinetic of spike specific T cells frequency in paired samples from KTRs and HPs at $21 \pm 4$ days (KTRs1 and HPs1) and $40 \pm 9$ days after the second dose of inactivated vaccine. (C) T cell responses targeted against SARS-CoV-2 NP in KTRs and HPs without vaccination or at $21 \pm 4$ days and $40 \pm 9$ days after the second dose of inactivated vaccine. (D) The kinetic of NP-specific T cell frequency in paired samples from KTRs and HPs at $21 \pm 4$ days (KTRs1 and HPs1) and 40 \pm 9 days after the second dose of inactivated vaccine. (E) T cell responses targeted against SARS-CoV-2 spike and NP in KTRs and HPs without vaccination or at $21 \pm 4$ days and $40 \pm 9$ days after the second dose of inactivated vaccine. (F) The kinetic of spike and NP- specific T cell frequency in paired samples from KTRs and HPs at $21 \pm 4$ days (KTRs1 and HPs1) and 40 \pm 9 days after the second dose of inactivated vaccine. The dotted line represents the cutoff value, which was calculated using mean \pm 2 standard deviations (SD) spike or NP-specific T cell frequency of HPs and KTRs without vaccination and COVID-19 history. 
Figure S1. Correlation between $\mathrm{T}$ cell immunity response and anti-SARS-CoV-2 antibody level in healthy participants (HPs) and kidney transplant recipients (KTRs) $21 \pm 4$ days after the second dose of inactivated vaccine. (A-D) The correlation between spike specific $\mathrm{T}$ cells frequency and anti-spike IgG (A), anti-receptor binding domain (RBD) IgG (B), RBD-Angiotensin I-converting enzyme 2 (ACE2) interaction blocking antibody (C), antinucleocapsid protein (NP) IgG (D) in HPs after the second dose of inactivated vaccine. (E-H) The correlation between NP-specific T cells frequency and anti-spike $\operatorname{IgG}(\mathrm{E})$, anti-receptor binding domain (RBD) IgG (F), RBDAngiotensin I-converting enzyme 2 (ACE2) interaction-blocking antibody (G), anti-nucleocapsid protein (NP) $\operatorname{IgG}$ (H) in HPs after the second dose of inactivated vaccine. (I-L) The correlation between spike specific $\mathrm{T}$ cells frequency and anti-spike IgG (I), anti-receptor binding domain (RBD) IgG (J), RBD-Angiotensin I-converting enzyme 2 (ACE2) interaction blocking antibody (K), anti-nucleocapsid protein (NP) $\operatorname{IgG}(\mathrm{L})$ in KTRs after the second dose of inactivated vaccine. (M-P) The correlation between NP specific T cells frequency and anti-spike IgG (M), anti-receptor binding domain (RBD) $\operatorname{IgG}(\mathrm{N})$, RBD-Angiotensin I-converting enzyme 2 (ACE2) interactionblocking antibody (O), anti-nucleocapsid protein (NP) IgG (P) in KTRs after the second dose of inactivated vaccine. 


\section{Figures}

\section{Figure 1}

Anti-SARS-CoV-2 antibodies in kidney transplant recipients (KTR) and healthy participants (HPs) at $21 \pm 4$ days (KTRs1 and HPs1) and 40 \pm 9 days (KTRs2 and HPs2) after the second dose of SARS-CoV-2 inactivated vaccine compared with KTRs or HPs without vaccination (KTRs0 and HPs0). (A) The optical density of anti-S1 IgG antibody in KTRs and HPs without vaccination and in $21 \pm 4$ days and $40 \pm 9$ days after the second dose of SARS-CoV-2 inactivated vaccine. (B) The optical density of anti-receptor binding domain (RBD) IgG antibody in KTRs and HPs without vaccination and in $21 \pm 4$ days and $40 \pm 9$ days after the second dose of SARS-CoV-2 inactivated vaccine. (C) The RBD-Angiotensin I Converting Enzyme 2 (ACE2) interaction blocking antibody in KTRs and HPs without vaccination and in $21 \pm 4$ days and $40 \pm 9$ days after the second dose of SARS-CoV-2 inactivated vaccine. (D) The optical density of anti-receptor binding domain (RBD) IgG antibody in KTRs and HPs without vaccination and in $21 \pm 4$ days and $40 \pm 9$ days after the second dose of SARS-CoV-2 inactivated vaccine. The horizontal dotted line indicates the cut-off value for positivity. The cut-off value was calculated using mean +3 standard deviation (SD) optical density of plasma samples from HPs and KTRs without vaccination.

\section{Figure 2}

T cell responses to pooled peptides from SARS-CoV-2 spike and nucleocapsid protein (NP) in kidney transplant recipients (KTR) and healthy participants (HPs) at 21 \pm 4 days (KTRs1 and HPs1) and 40 \pm 9 days (KTRs2 and HPs2) after the second dose of SARS-CoV-2 inactivated vaccine compared with KTRs or HPs without vaccination (KTRsO and HPs0). (A) T cell responses targeted against SARS-CoV-2 spike in KTRs and HPs without vaccination, or at $21 \pm 4$ days and $40 \pm 9$ days after the second dose of inactivated vaccine. (B) The kinetic of spike specific T cells frequency in paired samples from KTRs and HPs at $21 \pm 4$ days (KTRs1 and HPs1) and 40 \pm 9 days after the second dose of inactivated vaccine. (C) T cell responses targeted against SARS-CoV-2 NP in KTRs and HPs without vaccination or at $21 \pm 4$ days and $40 \pm 9$ days after the second dose of inactivated vaccine. (D) The kinetic of NP-specific T cell frequency in paired samples from KTRs and HPs at $21 \pm 4$ days (KTRs1 and HPs1) and $40 \pm 9$ days after the second dose of inactivated vaccine. (E) T cell responses targeted against SARS-CoV-2 spike and NP in KTRs and HPs without vaccination or at $21 \pm 4$ days and $40 \pm 9$ days after the second dose of inactivated vaccine. $(F)$ The kinetic of spike and NP-specific T cell frequency in paired samples from KTRs and HPs at $21 \pm 4$ days (KTRs 1 and HPs 1 ) and $40 \pm 9$ days after the second dose of inactivated vaccine. The dotted line represents the cut-off value, which was calculated using mean \pm 2 standard deviations (SD) spike or NPspecific T cell frequency of HPs and KTRs without vaccination and COVID-19 history. 


\section{Supplementary Files}

This is a list of supplementary files associated with this preprint. Click to download.

- figS1Supplymentalcorrelationship.jpg 